\title{
In-vivo imaging of oral squamous cell carcinoma by EGFR monoclonal antibody conjugated near-infrared quantum dots in mice
}

This article was published in the following Dove Press journal:

International Journal of Nanomedicine

I8 August 2011

Number of times this article has been viewed

Kai Yang

Fu-Jun Zhang

Hong Tang

Cheng Zhao

Yu-An Cao

Xiao-Qiang Lv

Dan Chen

Ya-Dong Li

Department of Oral and Maxillofacial Surgery, The First Affiliated Hospital,

Chongqing Medical University,

Chongqing, China
Correspondence: Kai Yang

Department of Oral and Maxillofacial Surgery, The First Affiliated Hospital,

Chongqing Medical University,

Chongaing 4000 I6, China

$\mathrm{Tel}+862389012569$

Fax +86 2389012569

Email cqfyyk@hotmail.com
Objectives: The purpose of this study was to investigate in-vivo visible imaging of oral squamous cell carcinoma (OSCC) by targeting epidermal growth factor receptor (EGFR) with near-infrared quantum dots.

Materials and methods: Quantum dots with an emission wavelength of $800 \mathrm{~nm}$ (QD800) were conjugated to monoclonal antibodies against EGFR, resulting in the probe designated as QD800-EGFR Ab. OSCC cell line (BcaCD885) expressing high levels of EGFR was transplanted subcutaneously into nude mice cheeks to develop an OSCC animal model. QD800-EGFR Ab containing 100 pmol equivalent of QD800 was intravenously injected into the animal model, and in-situ and in-vivo imaging of cheek squamous cell carcinoma was analyzed at 10 different time points.

Results and conclusion: In-vivo imaging and immunohistochemical examination of the tumors showed that intravenously injected QD800-EGFR Ab probe could bind EGFR expressed on BcaCD885 cells. Fluorescence signals of BcaCD885 cells labeled with QD800-EGFR Ab probe could be clearly detected, and these fluorescence signals lasted for 24 hours. The most complete tumor images with maximal signal-to-noise ratio were observed from 15 minutes to 6 hours after injection of the probe. To the best of the authors' knowledge, this is the first study that has obtained clear in-situ and in-vivo imaging of head and neck cancer by using QD800EGFR Ab probe. The authors conclude that the combination of near-infrared quantum dots that are highly penetrating for tissues with EGFR monoclonal antibody has promising prospects in in-vivo imaging of OSCC and development of personalized surgical therapies.

Keywords: oral cancer, head and neck cancer, near-infrared fluorescence, visual in-vivo imaging, epidermal growth factor receptor, nanotechnology

\section{Introduction}

Oral squamous cell carcinoma (OSCC) accounts for $90 \%$ of oral cancer, and currently surgery is one of the main treatments. ${ }^{1}$ The most difficult part in surgery is to correctly define the boundary of the tumor and precisely determine the regions for surgical resection in order to improve survival rate and quality of life. Visualized methods to detect the tumor cells during surgery are currently not available. Clinical doctors estimate the tumor boundary for surgical resection by experience and the changes of the tumor tissue texture, which results in a $40 \%$ failure rate of complete removal of head and neck cancer ${ }^{2,3}$ and greatly affects the survival rate of the patients. Therefore, development of methods for real-time identification of tumor cells during surgery and establishment of tailored surgical resection for each individual are one of the key factors in improving survival rate. 
Recently, quantum dots (QDs) were developed on the interdisciplinary advancement of nanotechnology, chemistry, and optics. The unique optical properties of QDs have shown promising prospects in the personalized surgical treatment for cancer patients. ${ }^{4,5}$ QDs are nanocrystals (with a diameter of $2-10^{\circ} \mathrm{nm}$ ) composed of elements belonging to group II-IV or group III-V. Compared with traditional fluorescence markers, QDs have narrow emission spectrum, wide excitation spectrum, high intensity of fluorescence, and good photochemical stability due to the quantum size and dielectric confinement effects. In addition, any emission spectrum from ultraviolet to near-infrared (or from blue to red) under the same excitation wavelength of light can be obtained by changing the particle size of QDs. ${ }^{6-8}$ These optical characteristics of QDs are not possessed by any of the current fluorescent probes, including a variety of organic fluorescent dyes and fluorescent proteins. Particularly, the fluorescence of recently developed QDs with an emission of near-infrared wavelength from $700 \mathrm{~nm}$ to $900 \mathrm{~nm}$ has strong penetration in human tissues, which is extremely suitable for visible in-vivo medical imaging. ${ }^{9-11}$ Currently, fluorescent probes have been developed by conjugating QDs with target molecules (eg, antibodies and peptides) and have been used for in-vivo visualization of cancer cells and tumor angiogenesis, ${ }^{4,12-14}$ sentinel lymph node detection, ${ }^{15,16}$ and imaging of drug targeting studies. ${ }^{17}$ These studies have demonstrated that excellent optical properties of QDs have promising prospects in visualization of cancer development and personalized therapies. Because QDs are made of toxic heavy metal materials, previous studies have shown that toxicity of the QDs mainly comes from a quick release of heavy metal (such as $\mathrm{Cd}$ ). In contrast, current biologically functionalized QDs have excellent biological compatibility and water solubility. More importantly, these biologically functionalized QDs do not have obvious side effects in humans at the required range of detection dosage and do not affect the growth, differentiation, and function of cells. . $^{7,818-20}$

Many studies have demonstrated that $90 \%$ of OSCCs and head and neck squamous cell carcinomas (HNSCCs) highly express epidermal growth factor receptor (EGFR). ${ }^{21,22}$ Specific targeting of EGFR by EGFR antibodies has been widely used for the treatment of OSCC and HNSCC..$^{22,23}$ Therefore, visual imaging by targeting EGFR has broad applicability for OSCC and HNSCC. Visualization of insitu and in-vivo imaging for OSCC and HNSCC by EGFR antibody conjugated QDs has not been reported. The authors of this paper attached EGFR monoclonal antibodies to QDs with a maximal emission wavelength of $800 \mathrm{~nm}$ to produce a probe designated as QD800-EGFR Ab. OSCC animal model was developed by transplanting nude mice subcutaneously with human buccal squamous cell carcinoma cell line (BcaCD885). Finally, QD800-EGFR Ab was intravenously injected into the animal model for in-situ and in-vivo imaging of OSCC. The results obtained in this study provided fundamental bases for personalized surgical treatment of OSCC and HNSCC by using QDs.

\section{Materials and methods Main equipment and reagents}

BcaCD885 was provided by West China College of Stomatology, Sichuan University (China). Qdot ${ }^{\mathbb{} 800}$ Antibody Conjugation Kits were purchased from Invitrogen (Carlsbad, CA). A recombinant human/mouse chimeric anti-EGFR monoclonal antibody and Histostain ${ }^{\mathrm{TM}}$-Plus kits were purchased from Beijing Zhong Shan Company (China). A laser scanning confocal microscope (TCS-SP5) was purchased from Leica (Germany). The Maestro In-Vivo imaging system was purchased from CRi Inc (Woburn, MA).

\section{Experimental animals}

Specific-pathogen-free level BALB/c (strain nu/nu) nude mice ( $\mathrm{n}=18$, female, $6-8$ weeks old, weight $20-25 \mathrm{~g}$ ) were purchased from the Experimental Animal Center of Chongqing Medical University. The mice were bred in constant temperature and humidity conditions. Feeds, beds, and drinking water were sterilized. All the experimental procedures were approved by the Animal Administration Committee of the Institute of Chongqing University.

\section{Preparation and purification of QD800-EGFR Ab probe}

Preparation of QD800-EGFR Ab probes was performed according to instructions of the Qdot Antibody Conjugation Kits. Briefly, $14 \mu \mathrm{L}$ of the dual function SMCC (succinimidyl4-(N-maleimidomethyl)cyclohexane-1-carboxylate) solution $(10 \mathrm{mM})$ was mixed with $125 \mu \mathrm{L}$ of QD800 solution $(4 \mu \mathrm{M})$ for 1 hour at room temperature. After 1-hour activation, the mixture was loaded onto an $\mathrm{NAP}^{\mathrm{TM}}-5$ (GE Healthcare, Little Chalfont, UK) column and a total of $500 \mu \mathrm{L}$ elution (Elution 1) was collected. A total of $6.1 \mu \mathrm{L}$ of dithiothreitol solution (1 M) was added to $300 \mu \mathrm{L}$ of EGFR monoclonal antibody $(1 \mathrm{mg} / \mathrm{mL})$. After 30 minutes of reduction reaction at room temperature, the dye indicators were added. The mixture was loaded onto an NAP-5 column, and a total of $500 \mu \mathrm{L}$ elution (Elution 2) was collected. Elution 1 and Elution 2 were mixed. After 1-hour coupling reaction at room 
temperature, $3 \mu \mathrm{L}$ of 2 -mercaptoethanol $(10 \mathrm{mM})$ was added for 30 minutes of inactivation. The coupled and inactivated solution obtained above was added into an ultra-filtration tube and was centrifuged for 15 minutes at $7000 \mathrm{rpm}$. The solution in the inner membrane of the ultra-filtration tube was collected and chromatography was conducted to obtain purified QD800-EGFR Ab probe. Finally, the concentration of QD800-EGFR Ab was calculated using the following formula: $\mathrm{A}=\varepsilon \mathrm{cl}$, where $\mathrm{A}$ represented absorbance, $\varepsilon$ represented the extinction coefficient, c represented the concentration, and 1 represented the optical path.

\section{Immunohistochemical detection of EGFR}

One milliliter of BcaCD885 cells at a concentration of $2 \times 10^{4}$ cells $/ \mathrm{mL}$ were seeded into each well of a 24 -well plate containing a glass coverslip. After 24 hours of culture, the glass coverslip was taken out and fixed with $4 \%$ paraformaldehyde for 30 minutes. Streptavidin peroxidase (SP) immunohistochemical staining was performed according to instructions of the Histostain-Plus kits. Briefly, the coverslips were incubated with $3 \% \mathrm{H}_{2} \mathrm{O}_{2}$ deionized water for 10 minutes, washed with distilled water, and soaked in phosphate buffered saline (PBS) for 5 minutes. Consequently, the coverslips were incubated with normal goat serum at room temperature for 15 minutes before EGFR monoclonal antibody (1:100) was added. After incubation overnight at $4^{\circ} \mathrm{C}$, the coverslips were washed with PBS three times and then biotin-labeled goat anti-rabbit immunoglobulin $\mathrm{G}$ and streptavidin conjugate of horseradish peroxidase were added. Finally, diaminobenzidine was added for color development. Negative control was performed by replacing the primary antibody with PBS. Positive control was provided by the Histostain-Plus kits. Appearance of brown-yellow granules within the cells indicated positive.

\section{In-vitro detection of the binding between EGFR and QD800-EGFR Ab probe}

BcaCD 885 cells at a concentration of $5 \times 10^{4}$ cells $/ \mathrm{mL}$ were seeded onto three $35 \mathrm{~mm}$-culture dishes with glass bottoms ( $1 \mathrm{~mL}$ in each dish). After 24 hours of culture, the cells were washed twice with PBS, and the cells were divided into three groups. The experimental group was added with $100 \mu \mathrm{L}$ of QD800-EGFR Ab probe (100 nM). The control group I was added to $100 \mu \mathrm{L}$ of QD800 (100 nM). The control group II was added to $200 \mu \mathrm{L}$ of EGFR monoclonal antibody ( $1 \mu \mathrm{g} / \mathrm{mL}$ ) for 2 hours of blocking. Subsequently, the cells were washed twice with PBS and then an equimolar amount of QD800-EGFR Ab probe was added to the experimental group. The cells in the three groups described above were incubated for 30 minutes at $37^{\circ} \mathrm{C}$ and then washed with PBS three times. Distribution of QD800 fluorescence within the cells was analyzed by confocal microscopy. All the experiments were repeated three times.

\section{In-vivo imaging of BcaCD885 cells by QD800-EGFR Ab probe in the OSCC mice models}

BcaCD885 cell suspension $\left(1 \times 10^{6}\right)$ in $0.1 \mathrm{~mL}$ PBS was transplanted subcutaneously into the right cheek of the nude mice $(\mathrm{n}=18)$ to develop the BcaCD885 cheek squamous cell carcinoma model. Three weeks post-injection, the maximal diameter of the tumor reached $0.8-1.1 \mathrm{~cm}$. The mice were divided into the experimental group $(n=6)$, control group I $(n=6)$, and control group II $(n=6)$. All the animals were anaesthetized by intraperitoneal injection of $2 \%$ pentobarbital (40 mg/kg). Subsequently, for the experimental group, $100 \mu \mathrm{L}$ of QD800-EGFR Ab probe containing $100 \mathrm{pmol}$ equivalent of QD800 was injected into the mice through the tail vein. For control group I, $100 \mu \mathrm{L}$ of QD800 (100 pmol) was injected. For control group II, $250 \mu \mathrm{L}$ of EGFR monoclonal antibody $(1 \mathrm{mg} / \mathrm{mL})$ was injected and 24 hours later an equimolar amount of QD800-EGFR Ab probe was injected. In-vivo imaging detection was performed 15 and 30 minutes, and 1, 2, 3, 4, 6, 8, 10, and 24 hours post-injection of QD800-EGFR Ab probe and QD800 using the Maestro in-vivo imaging system. Images were analyzed by Maestro 2.10.0 software.

\section{Cellular and histological examination of the BcaCD885 tumors}

Two mice from the experimental group, control group I, and control group II were euthanized 3 hours after injection of QD800-EGFR Ab probe and QD800. The remaining four mice from the experimental and control groups were euthanized 24 hours after QD800-EGFR Ab probe injection. The subcutaneous cheek tumors were removed from the euthanized mice and embedded in optimal cutting temperature compound. The embedded samples were frozen, and cryo-sections with a thickness of $7 \mu \mathrm{m}$ were continuously cut under $-20^{\circ} \mathrm{C}$ conditions. One of every two continuous sections was conducted with hematoxylin and eosin (H\&E) staining for observation of the tumor growth. The nucleus in another section was stained blue with Hoechst 33342 $(0.5 \mu \mathrm{g} / \mathrm{mL})$ for 5 minutes and then washed with PBS three times. Distribution of QD800 fluorescence in the tumor tissues was observed by confocal microscopy. 


\section{Statistical analysis}

The values were expressed as the mean \pm standard error. Statistical analysis was performed using Student's $t$-test. A $P$-value $<0.05$ was considered to be statistically significant. All statistical analyses were performed using the SPSS Statistics (IBM Corporation, Somers, NY) software package.

\section{Results}

\section{Preparation and purification of QD800-EGFR Ab probe}

Based on the extinction coefficient of QD800 at $550 \mathrm{~nm}$ $\left(\varepsilon_{550}=1.7 \times 10^{6}(\mathrm{~mol} / \mathrm{L})^{-1} \mathrm{~cm}^{-1}\right)$, the concentration of the purified QD800-EGFR Ab probe was calculated to be $2 \mu \mathrm{M}$.

\section{QD800-EGFR Ab probe specifically binds EGFR of BcaCD885 cells in vitro}

SP immunohistochemical analysis indicated that EGFR was expressed on the membrane and in the cytoplasm of BcaCD855 cells (Figure 1). Direct immunofluorescence examination with QD800-EGFR Ab probe showed that red fluorescence was present on the membrane of BcaCD885 cells in the experimental group. In contrast, red fluorescence was not observed in the BcaCD885 cells in control groups I or II (Figure 2). These results demonstrated that QD800-EGFR $\mathrm{Ab}$ probe cannot label EGFR of BcaCD885 cells that had been blocked by EGFR antibody. In contrast, QD800-EGFR $\mathrm{Ab}$ probe can recognize and bind efficiently with unblocked EGFR of BcaCD885 cells in vitro.

\section{Visible in-situ and in-vivo imaging of OSCC by QD800-EGFR Ab probe}

In-vivo imaging analysis showed that the size of the fluorescence signal equivalent to the size of the tumors can be detected 15 minutes after injection of QD800-EGFR Ab probe in the experimental group. The size of fluorescent
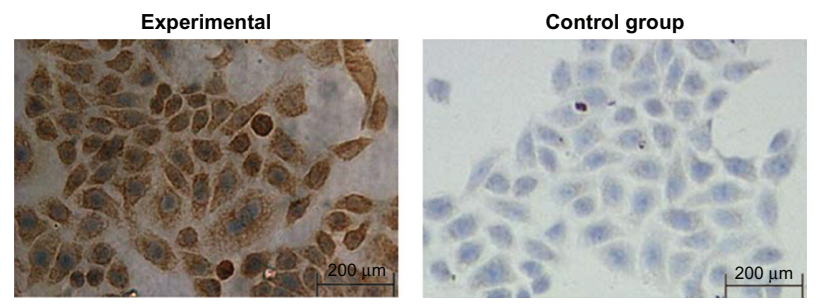

Figure I Immunohistochemical examination of EGFR expression on the membrane and in the cytoplasm of BcaCD885 cells in the experimental group and control group (the primary antibody was replaced by PBS) $(S P \times 200)$.

Abbreviations: $\mathrm{BcaCD} 885$, human buccal squamous cell carcinoma cell line; EGFR, epidermal growth factor receptor; PBS, phosphate buffered saline; SP, streptavidin peroxidase.

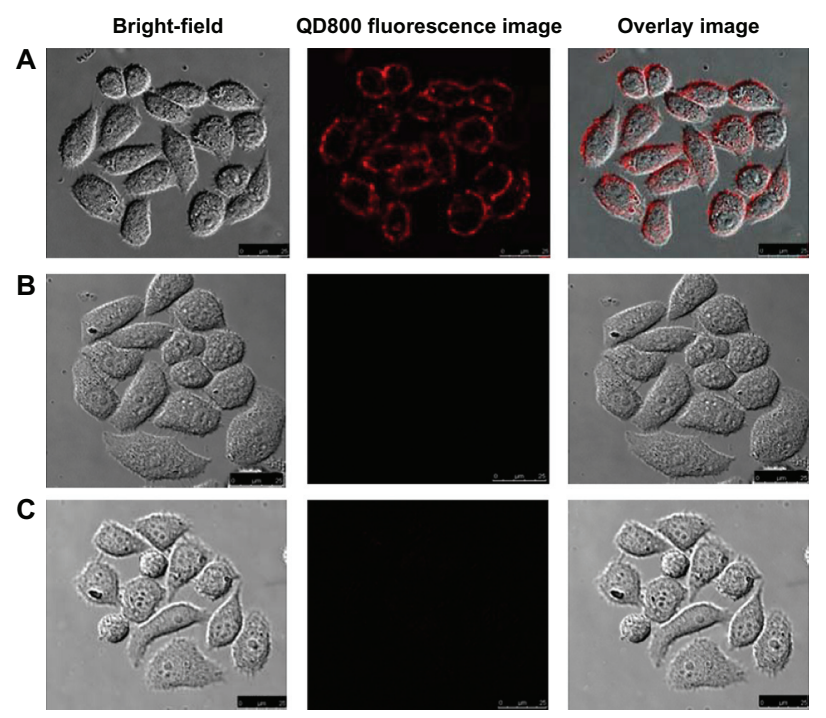

Figure 2 In-vitro labeling of BcaCD885 cells with QD800-EGFR Ab probe. Detection of the fluorescence was conducted after 30 minutes of labeling. The excitation wavelength was $405 \mathrm{~nm}$, and the emission wavelength was 750-800 nm. (A) Experimental group: the cells were labeled with QD800-EGFR Ab probe $(100 \mathrm{nM})$ at $37^{\circ} \mathrm{C}$ for 30 minutes. (B) Control group I: the cells were added with QD800 (100 nM) at $37^{\circ} \mathrm{C}$ for 30 minutes. (C) Control group II: the cells were blocked with $200 \mu \mathrm{L}$ of EGFR monoclonal antibody $(\mathrm{I} \mu \mathrm{g} / \mathrm{mL})$ for 2 hours before addition of QD800-EGFR Ab probe $(100 \mathrm{nM})$ at $37^{\circ} \mathrm{C}$ for 30 minutes.

Abbreviations: $\mathrm{Ab}$, antibodies; $\mathrm{BcaCD} 885$, human buccal squamous cell carcinoma cell line; EGFR, epidermal growth factor receptor; QD800, quantum dots with a maximal emission wavelength of $800 \mathrm{~nm}$.

signal persisted for 6 hours post-injection, and after 8 hours the size of the signal began to become significantly smaller (Figure 3A). Twenty-four hours after the probe injection, the fluorescent signal was very weak (Figure 3A). The signal-to-noise ratio (the fluorescence intensity ratio between tumor and background) was high from 15 minutes to 6 hours post-injection. This signal-to-noise ratio began to decrease significantly after 8 hours and was close to the baseline level after 24 hours (Figure 3C). In control group I, QD800 was not conjugated with EGFR Ab. In control group II, EGFR was blocked before injection of QD800-EGFR Ab probe, and thus only a very weak fluorescence signal was detected on the tumor sites 15 minutes post-injection in control groups I and II. This weak signal could be due to the nonspecific phagocytosis of QD800-EGFR Ab probe and QD800 by BcaCD885 cells. However, the fluorescence signal above the background level was never detected 30 minutes to 24 hours post-injection in control groups I and II (Figure 3B).

\section{Cellular and histological detection of BcaCD885 tumors}

$\mathrm{H} \& \mathrm{E}$ staining of the tumor sections from the mice 3 and 24 hours post-injection showed a large amount of tumor cells, and the tumors grew well (Figure 4). The nucleus of tumor 
A
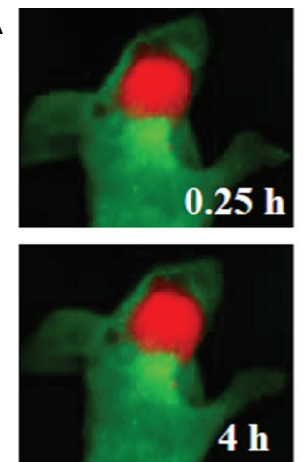

B

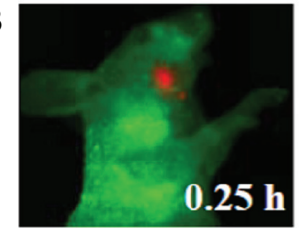

C

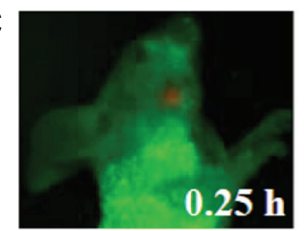

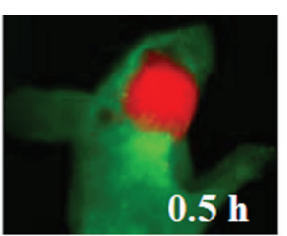
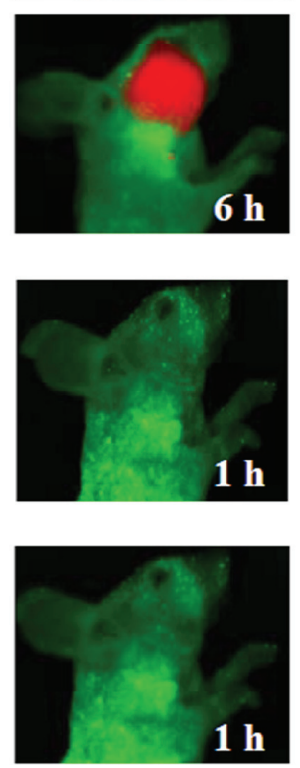
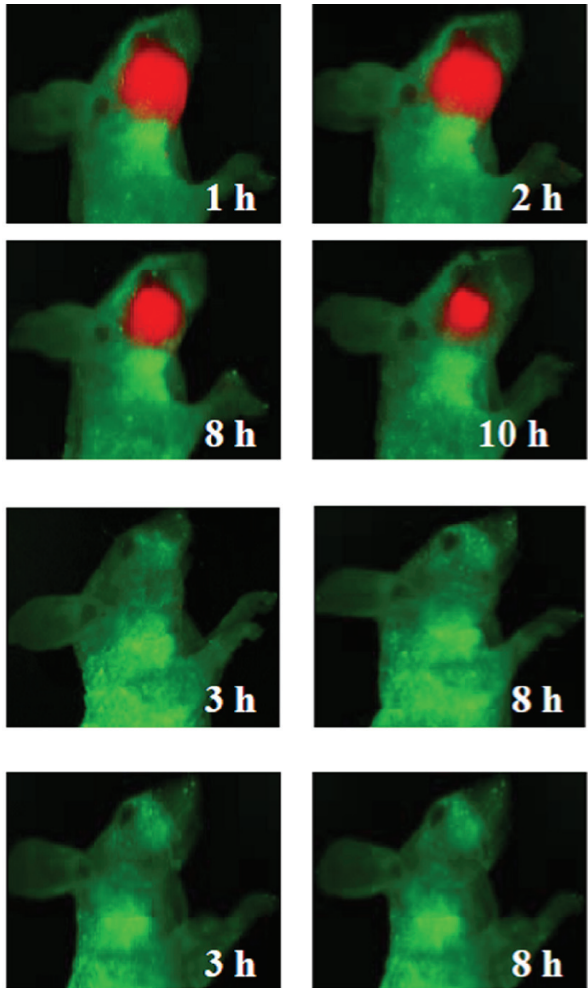
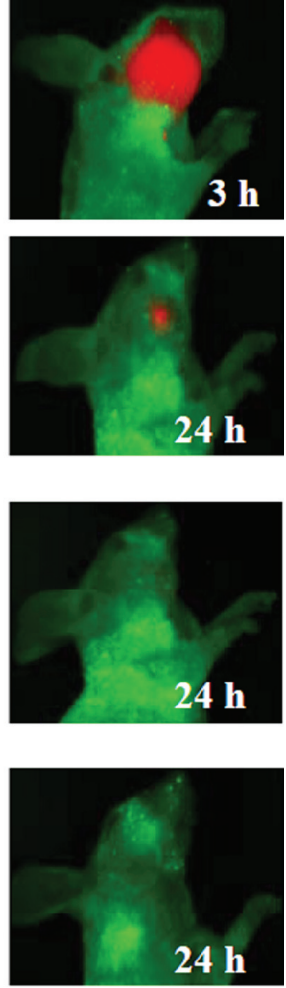

D

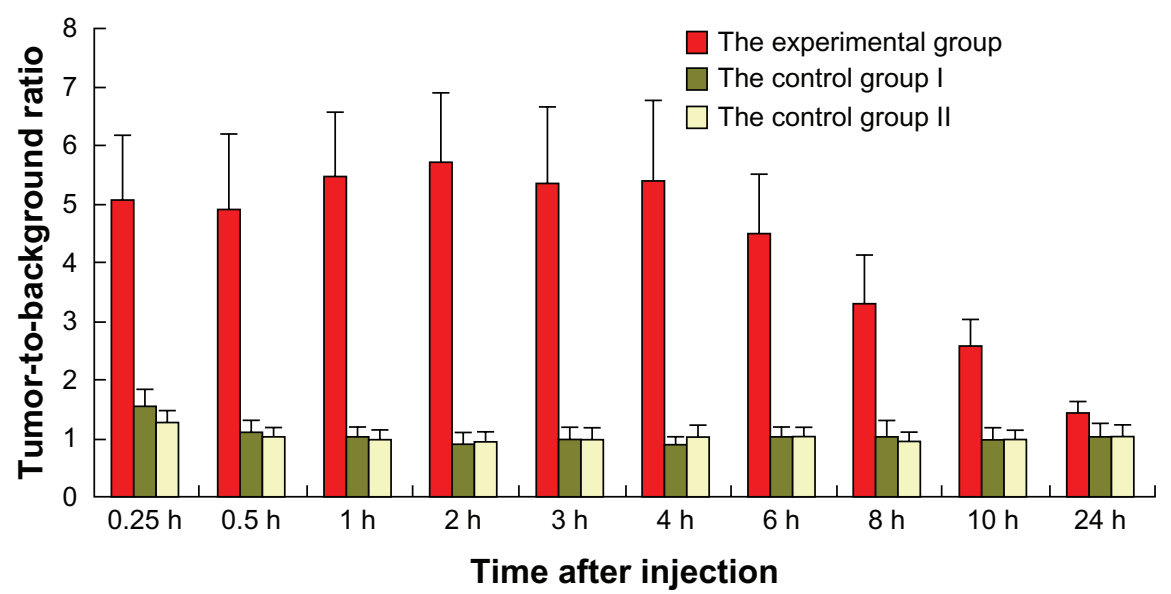

Figure 3 Visible imaging of BcaCD885 cells in the cheek squamous cell carcinoma animal model by QD800-EGFR Ab probe at different times (excitation/emission $630 / 800 \mathrm{~nm}$; exposure time $50 \mathrm{~ms}$; collection time 10 seconds, $2 \times 2$ Binning $1024 \times 1024$ pixels). (A) Visible images of BcaCD885 cheek squamous cell carcinoma mice after intravenous injection with QD800-EGFR Ab probe containing 100 pmol equivalent of QD800 in the experimental group. (B) Visible images of BcaCD885 cheek squamous cell carcinoma mice after intravenous injection with 100 pmol QD800 in control group I. (C) Visible images of BcaCD885 cheek squamous cell carcinoma mice in control group II ( $250 \mu \mathrm{L}$ of EGFR monoclonal antibody $(\mathrm{I} \mathrm{mg} / \mathrm{mL})$ was injected, and 24 hours later QD800-EGFR Ab probe containing 100 pmol equivalent of QD800 was injected). (D) The signal-to-noise ratio in the BcaCD885 squamous cell carcinoma model after different times of probe injection in the experimental group.

Abbreviations: Ab, antibodies; BcaCD885, human buccal squamous cell carcinoma cell line; EGFR, epidermal growth factor receptor; QD800, quantum dots with a maximal emission wavelength of $800 \mathrm{~nm}$.

sections was stained blue by Hoechst 33342 under confocal microscope. QD800 fluorescence signal was not detected in the tumor section of the mice in control groups I and II 3 and 24 hours post-injection. In contrast, in the experimental group, QD800 fluorescence signal was detected outside of most of the nucleus in the tumor section of the mice 3 hours post-injection (Figure 5). Twenty-four hours post-injection, a fluorescence signal was detected only outside a small number of nuclei (Figure 5). The fluorescence signal was distributed in the cytoplasm and on the membrane. These results indicated that QD800-EGFR Ab probe can specifically target and bind EGFR of BcaCD885 cells. 
$3 \mathrm{~h}$
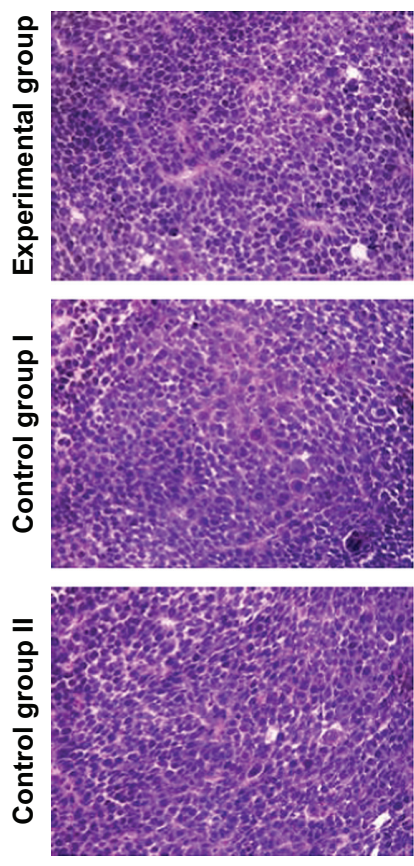

$24 \mathrm{~h}$
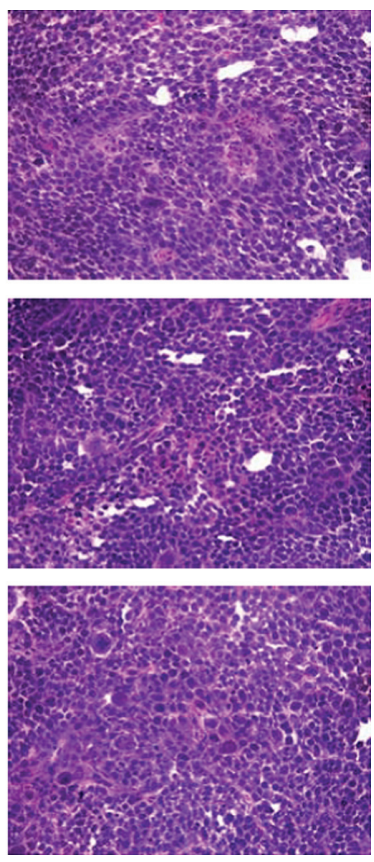

Figure $4 \mathrm{H} \& \mathrm{E}$ staining of tumor section from the experimental group (intravenous injection of QD800-EGFR Ab probe), control group I (intravenous injection of QD800), and control group II (preinjection of EGFR monoclonal antibody 24 hours before QD800-EGFR Ab probe injection) of BcaCD885 squamous cell carcinoma 3 and 24 hours after probe injection $(\times 200)$.

Abbreviations: Ab, antibodies; $\mathrm{BcaCD} 885$, human buccal squamous cell carcinoma cell line; EGFR, epidermal growth factor receptor; H\&E, hematoxylin and eosin; QD800, quantum dots with a maximal emission wavelength of $800 \mathrm{~nm}$.

\section{Discussion}

Development of methods to visualize tumor cells during surgery and determine the precise boundary of the tumors for personalized surgical resection is the key to improve the survival rate of cancer patients. Traditional methods (eg, computed tomography $[\mathrm{CT}]$ and magnetic resonance imaging [MRI]) that can provide good imaging detection of tumors are not suitable for visible detection of tumor cells during surgery. Optical examination is clinically the safest, simplest, and most economical method. QDs have unique optical properties as mentioned earlier. More importantly, QDs can be easily modified and conjugated by other biological molecules. As nanoparticles, QDs with good photochemical stability can easily penetrate tumor angiogenesis and access cancer cells. Therefore, QDs have shown unique advantages in the surgical treatment of individual cancer patients. ${ }^{6-11}$

In this study, we intravenously injected QD800-EGFR Ab probe to target EGFR, which is highly expressed by OSCC. In-vivo imaging and tumor immunohistochemical analysis showed that QD800-EGFR Ab probe can specifically bind BcaCD885 cells with high level expression of EGFR. Thus, the fluorescence of QD800 can represent the presence of

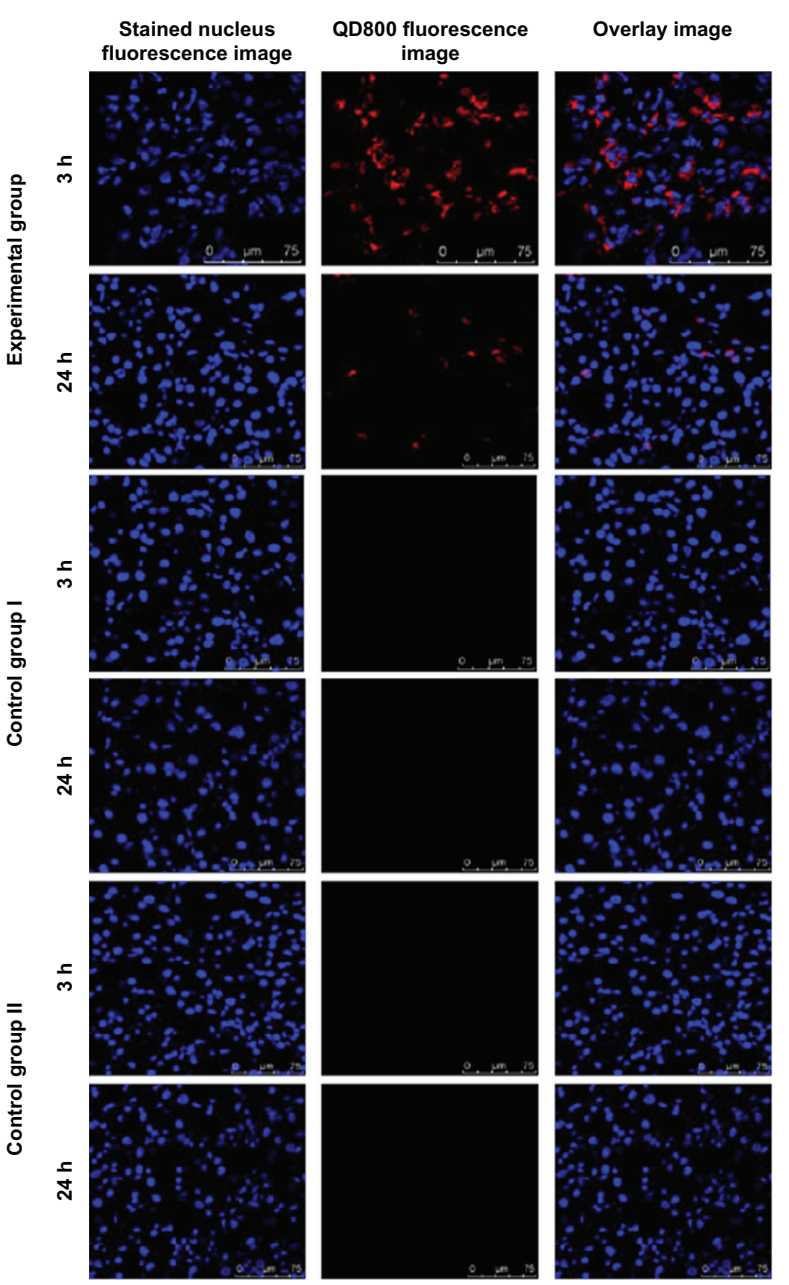

Figure 5 Laser scanning confocal microscope analysis of tumor section from the experimental group (intravenous injection of QD800-EGFR Ab probe), control group I (intravenous injection of QD800), and control group II (preinjection of EGFR monoclonal antibody 24 hours before QD800-EGFR Ab probe injection) of BcaCD885 squamous cell carcinoma 3 and 24 hours after probe injection. Nucleus was stained by Hoechst 33342.

Abbreviations: $\mathrm{Ab}$, antibodies; $\mathrm{BcaCD} 885$, human buccal squamous cell carcinoma cell line; EGFR, epidermal growth factor receptor; QD800, quantum dots with a maximal emission wavelength of $800 \mathrm{~nm}$.

$\mathrm{BcaCD} 885$ cells and can be visualized from outside of the body; toxicity of the QDs in the mice was not observed in this study. The complex metabolic process of QD800-EGFR $\mathrm{Ab}$ probe in the body is currently unknown. However, we obtained the most complete fluorescence images and highest signal-to-noise ratio 15 minutes to 6 hours post-injection, indicating that the optimal time period for personalized surgery is between 15 minutes and 6 hours after injection of QD800-EGFR Ab probe. Previous studies ${ }^{4,10}$ also show that $10^{4}$ cancer cells can be visibly detected after QD800 labeling in the presence of skin barrier. This sensitivity was 100-fold higher than that of CT and MRI. In addition, the sensitivity can be further enhanced when the tumor is exposed during the surgery. Gao et al predicted that in-vivo visible imaging 
can detect as low as 10-100 QD-labeled cancer cells. ${ }^{24}$ With the continuous advancement in imaging techniques, and synthesis of QDs with stronger penetration abilities for tissues, the authors of this paper believe that visible imaging detection can reach sensitivity at single-cell level under the wound exposure condition. In the future, clinical doctors will only need to wear a very small excitation source and nearinfrared light receiver to perform personalized and tailored surgical removal of real tumors.

\section{Acknowledgments}

This study was supported by the National Natural Science

Foundation of China (No: 30872925).

\section{Disclosure}

The authors declare that they have no competing interests.

\section{References}

1. Kademani D, Bell RB, Brian, et al. Oral and maxillofacial surgeons treating oral cancer: a preliminary report from the American Association of Oral and Maxillofacial Surgeons Task Force on Oral Cancer. J Oral Maxillofac Surg. 2008;66(10):2151-2157.

2. Woolgar JA, Triantafyllou A. A histopathological appraisal of surgical margins in oral and oropharyngeal cancer resection specimens. Oral Oncol. 2005;41(10):1034-1043.

3. Rosenthal EL, Kulbersh BD, Duncan RD, et al. In vivo detection of head and neck cancer orthotopic xenografts by immunofluorescence. Laryngoscope. 2006;116(9):1636-1641.

4. Yang K, Cao YA, Shi C, et al. Quantum dot-based visual in vivo imaging for oral squamous cell carcinoma in mice. Oral Oncol. 2010;46(12):864-868.

5. Michalet X, Pinaud FF, Bentolila LA, et al. Quantum dots for live cells, in vivo imaging, and diagnostics. Science. 2005;307(5709):538-544.

6. Walling MA, Novak JA, Shepard JR. Quantum dots for live cell and in vivo imaging. Int J Mol Sci. 2009;10(2):441-491.

7. Lin S, Xie X, Patel MR, et al. Quantum dot imaging for embryonic stem cells. BMC Biotechnol. 2007;7:67.

8. Sun D, Yang K, Zheng G, Li Z, Cao Y. Study on effect of peptideconjugated near-infrared fluorescent quantum dots on the clone formation, proliferation, apoptosis, and tumorigenicity ability of human buccal squamous cell carcinoma cell line BcaCD885. Int J Nanomedicine. 2010;5:401-405.
9. Jiang W, Singhal A, Kim BYS, et al. Assessing near-infrared quantum dots for deep tissue, organ, and animal imaging applications. J Assoc Lab Autom. 2008;13:6-12.

10. Cao Y, Yang K, Li Z, Zhao C, Shi C, Yang J. Near-infrared quantum-dot-based non-invasive in vivo imaging of squamous cell carcinoma U14. Nanotechnol. 2010;21(47):475104.

11. Aswathy RG, Yoshida Y, Maekawa T, Kumar DS. Near-infrared quantum dots for deep tissue imaging. Anal Bioanal Chem. 2010; 397(4):1417-1435.

12. Tada $\mathrm{H}$, Higuchi $\mathrm{H}$, Wanatabe TM, Ohuchi N. In vivo real-time tracking of single quantum dots conjugated with monoclonal anti-HER2 antibody in tumors of mice. Cancer Res. 2007;67:1138-1144.

13. Cai W, Shin DW, Chen K, et al. Peptide-labeled near-infrared quantum dots for imaging tumor vasculature in living subjects. Nano Letters. 2006;6:6669-6676.

14. Gao J, Chen K, Xie R, et al. In vivo tumor-targeted fluorescence imaging using near-infrared non-cadmium quantum dots. Bioconjugate Chem. 2010;21(4):604-609.

15. Frangioni JV, Kim SW, Ohnishi S, Bawendi MG. Sentinel lymph node mapping with type II quantum dots. Methods Mol Biol. 2007;374:147-159.

16. Ballou B, Ernst LA, Andreko S, et al. Sentinel lymph node imaging using quantum dots in mouse tumor models. Bioconjugate Chem. 2007;18:389-396.

17. van Vlerken LE, Amiji MM. Multi-functional polymeric nanoparticles for tumour-targeted drug delivery. Expert Opin Drug Deliv. 2006;3(2):205-216.

18. Yang K, Li Z, Cao Y, Yu X, Mei J. Effect of peptide-conjugated near-infrared fluorescent quantum dots (NIRF-QDs) on the invasion and metastasis of human tongue squamous cell carcinoma cell line Tca8113 in vitro. Int J Mol Sci. 2009;10(10):4418-4427.

19. Zrazhevskiy P, Gao X. Multifunctional quantum dots for personalized medicine. Nano Today. 2009;4(5):414-428.

20. Li ZG, Yang K, Cao YA, Zheng G, Sun DP, Zhao C. In vivo study of the effects of peptide-conjugated near-infrared fluorescent quantum dots on the tumorigenic and lymphatic metastatic capacities of squamous cell carcinoma cell line Tca8113 and U14. Int J Mol Sci. 2010;11(4):1413-1422.

21. Rogers SJ, Harrington KJ, Rhys-Evans P, O-Charoenrat P, Eccles SA. Biological significance of c-erbB family oncogenes in head and neck cancer. Cancer Metastasis Rev. 2005;24(1):47-69.

22. Kalyankrishna S, Grandis JR. Epidermal growth factor receptor biology in head and neck cancer. J Clin Oncol. 2006;24(17):2666-2672.

23. Perri F, Longo F, Ionna F, Caponigro F. Recent results of cetuximab use in the treatment of squamous cell carcinoma of the head and neck. Onco Targets Ther. 2009;2:243-250.

24. Gao X, Cui Y, Levenson RM, Chung LW, Nie S. In vivo cancer targeting and imaging with semiconductor quantum dots. Nat Biotechnol. 2004;22(8):969-976.
International Journal of Nanomedicine

\section{Publish your work in this journal}

The International Journal of Nanomedicine is an international, peerreviewed journal focusing on the application of nanotechnology in diagnostics, therapeutics, and drug delivery systems throughout the biomedical field. This journal is indexed on PubMed Central, MedLine, CAS, SciSearch ${ }^{\circledR}$, Current Contents ${ }^{\circledR} /$ Clinical Medicine,

\section{Dovepress}

Journal Citation Reports/Science Edition, EMBase, Scopus and the Elsevier Bibliographic databases. The manuscript management system is completely online and includes a very quick and fair peer-review system, which is all easy to use. Visit http://www.dovepress.com/ testimonials.php to read real quotes from published authors. 